\title{
SIMULATION BASED DECISION SUPPORT SYSTEM TO DETERMINE PRODUCTION QUANTITY FOR A LOW SHELF LIFE PRODUCT
}

Mr. Amit Kumar

ITC Infotech India Ltd.

Cox Town, Bangalore 560005

amit.m@itcinfotech.com
Mr. Krishna Chaitanya Pappu

ITC Infotech India Ltd.

Cox Town, Bangalore 560005

krishnachaitanya.pappu@itcinfotech.com

\section{Mr. Sumit Kumar}

ITC Infotech India Ltd.

Cox Town, Bangalore

kumar.sumit@itcinfotech.com

\begin{abstract}
In this paper we show how a Decision Support System (DSS) using simulation of a low shelf life pharmaceutical product's supply chain was created. The main technique that determines the production quantity is based on a simulation-based optimization approach. The system allows the planners to quickly see the impact of various strategies and changes in policies and take the best decision.

The medicine is for a critical disease and hence the service level must be maintained upwards of 99.9\%. To maintain this high service level, especially when the shelf life of the medicine is three weeks is a challenge for any supply chain. The tradeoff between service level and inventory scrapping cost (due to medicine expiry) is optimized using a simulation-based optimization approach.
\end{abstract}

Keywords: Discrete event simulation modelling, Anylogic, Inventory optimization, Low shelf life

\section{INTRODUCTION}

The traditional optimization methods such as mixed integer programming, dynamic programming are well suited for strategic and tactical level decision making. Whereas for many operational and tactical level decision making in a complex real-world scenario, optimization methods are sometimes not practical. This is mainly because of the limitations of deterministic optimization methods of not being able to consider variability or uncertainty inherent in the system. Another challenge is that many of the real problems are combinatorial optimization problems which are NP-Hard. In such a situation simulation becomes a viable tool for problem solving. Hybrid models that combine simulation and optimization are also becoming more popular and demonstrating their efficacy (Muhammed Ordu et.al., 2020)

The client is a world leading pharmaceutical company with headquarters in Europe. For a medicine which treats a terminal illness, the shelf life of the medicine is as low as 21 days. Since this is a critical medicine delivered directly to the hospitals the service level needs to be maintained at upwards of $99.9 \%$. The obvious risk is of write-offs. The client was facing the challenge of high scrapping due to medicine expiry.

We delivered a solution based on the Anylogic simulation software (Borshchev A and Grigoryev I, 2020) that would take multiple uncertainties into account and simulate the supply chain with these uncertainties while optimizing the inventory to achieve the desired service level and minimizing the write-offs cost. 
The organization of this paper is as follows. In section 2 we give a literature review for similar problems. In section 3 we give a detailed description of the problem. In section 4 we explain our solution methodology and in section 5 we share the results of our work.

\section{LITERATURE REVIEW}

The management of the perishable products supply chain has gained traction in the past decade (Amorim et al., 2011). There has been research to develop sustainable solutions to handle product perishability, in the areas of manufacturing, storage, packaging, and transport operations (Accorsi et al., 2017). Most of the studies have focused on a common objective of improving the overall supply chain performance.

Looking into the area of manufacturing of perishable products, most of the research was focused on addressing the issues like supply fluctuations, improving quality, improving productivity and production technologies with a little attention to wastage at production (Yared Lemma et al., 2014)

When it comes to inventory decisions, the models related to inventory, which decays in terms of its utility over time are analysed. Zhaotong Lian and Liming Liu (2001) proposed a heuristic approach for continuous review of perishable inventory systems. QinglinDuanT and WarrenLiao (2013) proposed a simulation optimization methodology for inventory management of perishable products by considering order-up-to policy approach for handling highly perishable products. Xiaojun Wang and Dong Li (2012) proposed a dynamic pricing model to reduce food spoilage waste and maximise profit through a pricing approach based on dynamically identified shelf life. While the research on inventory models of deteriorating items has increased greatly over the last years, there is still some limited attention to inventory loss reduction decisions of perishable products in uncertain environments

This paper focuses on loss reduction in perishable product supply chains by determining the quantity of production to minimize the inventory scrapping cost. We have used simulation-based optimization approach to create a DSS.

\section{PROBLEM DESCRIPTION}

The supply chain for the medicine looks as shown in Figure 1. There are two manufacturing facilities, two distribution centers and numerous hospitals where the medicine should reach. Most of the demand in Europe, African and part of the Asian regions is served from Europe, with one of the manufacturing facilities and distribution centers located in Europe, while the rest of demand from US, Canada and rest of the Asian regions are served from manufacturing facility and distribution centers located in US.

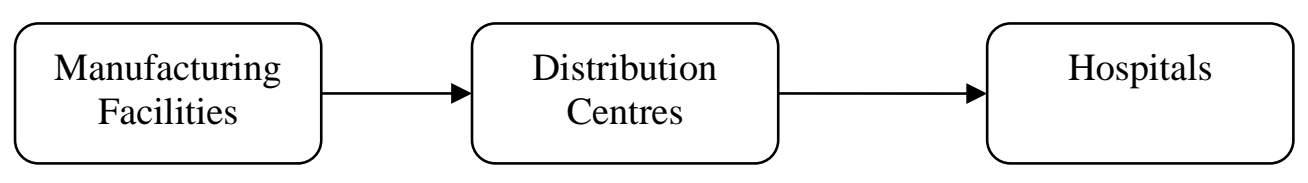

Figure 1 Supply chain overview

Since the shelf life is only three weeks, the production is done based on forecast and then the product is pushed to the distribution centers. The flow from distribution center to hospital happens on actual order. The limited shelf life of the medicine is due to natural decay of the medicine. As an example, a 10-day old medicine can be used for a patient with weight around $100 \mathrm{~kg}$, whereas a 15-day old medicine can be used for a patient with weight less than $70 \mathrm{~kg}$. After 21 days the medicine can only be used for patients below $45 \mathrm{~kg}$, and hence we consider them expired (the numbers mentioned are only for illustration).

The manufacturing facility produces various other products also in the same production line on a production campaign basis. The production campaign details are known in advance. We know the days in a week (typically one or two days) when the concerned medicine is produced. The decision support system needs to output what quantity should be produced on those days. The production capacity is enough and hence no production capacity constraint is necessary.

The medicine needs to be shipped to the hospital. For the purpose of lead time calculation for planning each country is divided into one or more delivery zones depending on country size. The lead 
time information is maintained and used at the zone level. Each hospital is assigned to a zone. Forecast is done at a week and zone level.

There are various sources of variability in this supply chain. The production is done based on forecast which comes with a forecast error. The weight of patient and the weekday of the treatment cannot be determined in advance and hence have uncertainty. The production can have batch failures. The transportation lead times have variability.

\section{SOLUTION METHODOLOGY}

The Enterprise Resource Planning (ERP) system provides master data such as data of the Stock Keeping Units (SKU), mapping of which SKU can be supplied in which country, historic lead times from manufacturing facility to Distribution Centre (DC) and DC to delivery zones. Transactional data such as proposed lead times for both legs of distribution based on weekday of shipment, production campaigns, current inventory snapshot, forecasts are also read from ERP database. In Figure 2 we provide an overview of the technical architecture of our solution.

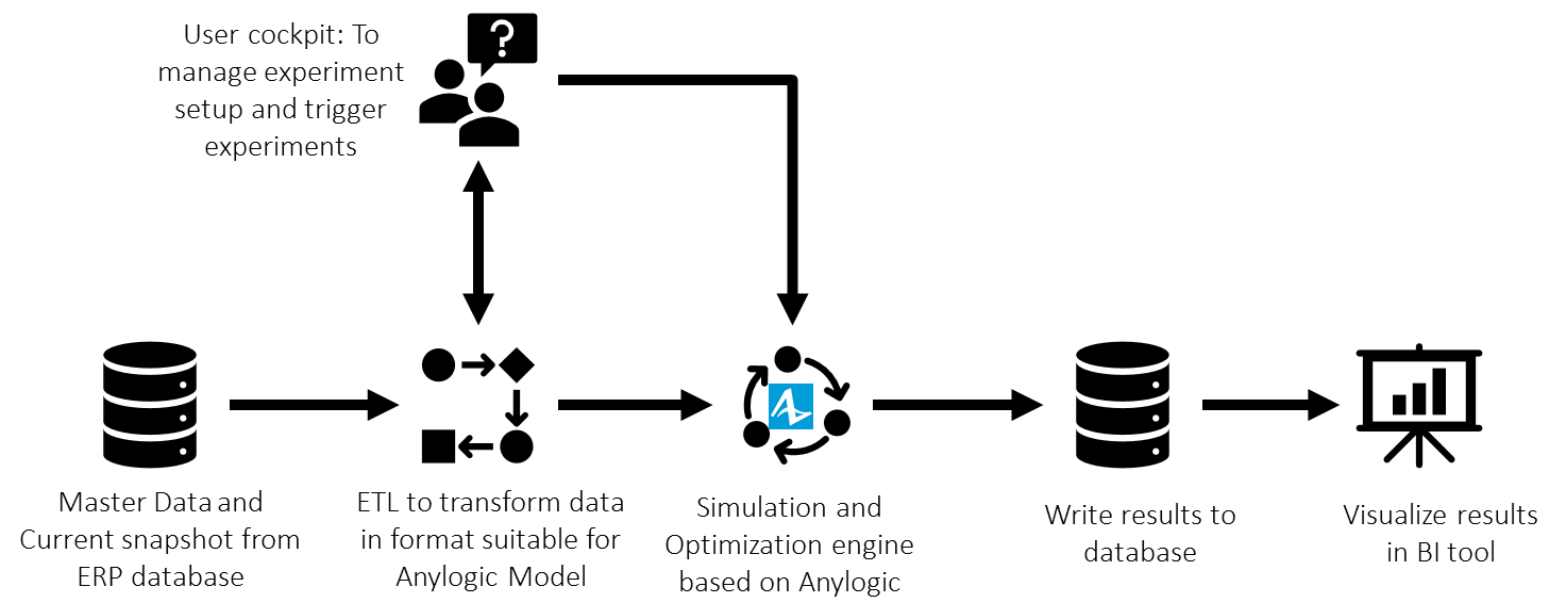

Figure 2 Technical architecture of solution

User Cockpit (excel based UI) facilitates users with options to choose the horizon of simulation, whether to run in pure simulation mode or digital twin mode. Digital twin mode will initialize experiment with current snapshot information whereas pure simulation model will use a warmup period. The length of the warmup period is also controlled from the user cockpit. Other options include choosing simulation or simulation + optimization, consider or adjust forecast biases and uncertainties, choose among production and distribution strategies etc. Once user is satisfied with the settings of the experiment, he can trigger the experiment from user cockpit itself. This will first launch the Extract Transform and Load (ETL) phase which will pull data from ERP and transform it and write data into Anylogic database in a form that the model will use. Once the ETL phase is complete, the ETL will communicate this back to cockpit logic and now cockpit will trigger the simulation experiment. Additionally, ETL will also perform all the checks and validations on the data. If any errors or warnings are found it will write the messages to the log file.

In Simulation phase, the simulation model runs the scenarios chosen by the user and on completion pushes the output results to database table.

The database loads the data from tables to views required for visualisations in a Business Intelligence (BI) Tool. The dashboard provides Key Performance Indicators (KPIs) visualisation to analyse the demands, production plans, write offs, compare statistics across the different scenarios and identify the parameter values that provides optimal write-offs and service level. 


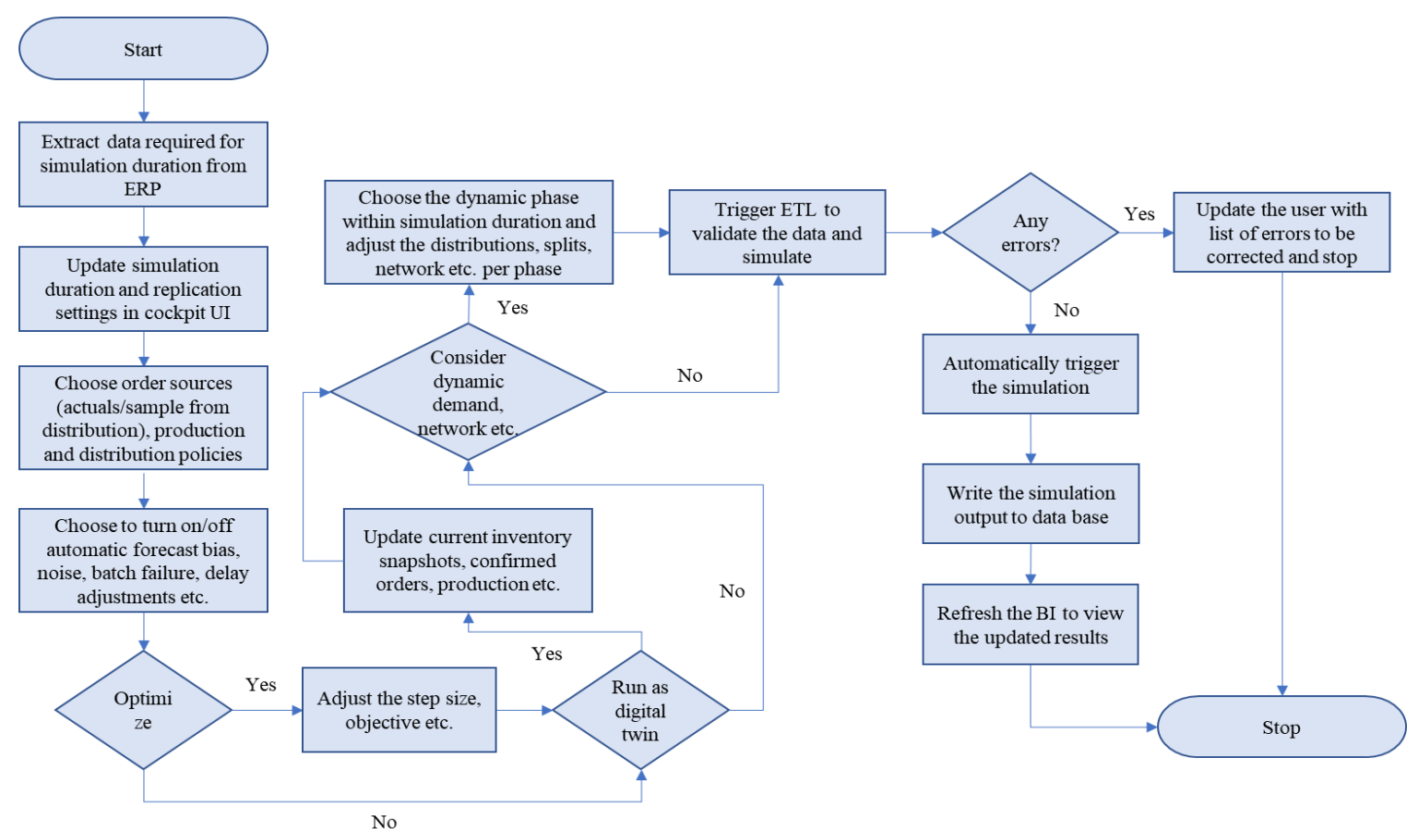

Figure 3 Solution flow chart

\subsection{Overview of production policy}

The production policy determines the quantity to be produced on production days by considering the inputs like production campaigns, confirmed orders, starting inventory, demand forecasts, zone splits(historical split of a country's demand at delivery zone level), weekday splits (historical split of demand at weekday level at hospitals), picking priorities etc. The planning is done on a weekly basis to determine the quantity to be produced for each SKU in the corresponding weeks. Different policies or production logic were tried and with each policy certain parameters were chosen to fine tune the policy using simulation-based optimization approach.

While the confirmed orders have all the required details like patient weights, days of treatment, zones of treatment etc. the forecasts are converted into daily orders by assigning these weights, treatment days, zones etc. through probabilistic sampling.

On the supply side, the variables that have impact on the service level have been determined by running the simulation over multiple replications by fixing the other attributes. The variables that had major impact on the service level have been included for supply side planning

The supply-side uncertainties considered are like batch failures, shipment delays, shipment damages etc. and demand side uncertainties like demand surges, high patient weights etc. are considered while planning for the safety quantity in addition to the cycle quantity.

The orders generated from forecasts are netted using available inventory first, followed by allocating them to the freshest production campaign to maintain highest serviceability and taking care of some demand side uncertainties like patient weights and treatment days. The production quantity per campaign is finalised by aggregating all the orders planned against each campaign and the quantities aggregated against the campaigns from immediate next week are frozen and sent for production. Hence, on the Monday of Week0 (current week), we plan and freeze the production in Week1 (next week).

The safety quantity represents the extra days of coverage that will be kept. This is determined at a country level and these are the parameters that will be optimized to fine tune the production policy selected.

\subsection{Stock optimization and scenario planning}

By changing the settings in user cockpit, we can perform optimizations as well as simulate and evaluate multiple scenarios. Some of these scenarios are explained below: 
Stock optimization: Users can select among few different production policies and for each policy determine safety quantity at a country level that provides minimum write-offs while maintaining the highest service level.

Validate production plans: Users can validate the production plans by generating the demand sampled from a distribution and simulating it over multiple replications and evaluating the output. The demand will have the details of delivery zone, patient weight and treatment date.

Switch on/off uncertainties: Users can turn on/off multiple uncertainties while planning for production to obtain a robust output. Ex: consider/adjust lead time distributions, consider/adjust production batch failures, consider/adjust shipment delays, consider/adjust forecast bias, consider/adjust forecast noise etc.

Future projections: Users can simulate and project the sales into long term/short term future by using dynamic demand distributions for different periods in future catering to seasonality/trends in demand.

Evaluate risks by dynamic network changes: Users can evaluate dynamic network changes by switching the production and distribution sources and destination zones during special weeks/manufacturing facility closures etc. for specific time periods.

\subsection{Simulation based optimization approach}

The general framework of a simulation-based optimization approach is provided by C. Almeder and M. Preusser (2007). We use a similar approach. The Anylogic simulation software also provides an optimization engine based on OptQuest. We use the OptQuest engine as a black box for optimization. Doing multiple simulation runs and analysing the output of these runs we can see the impact of variation. In each simulation run different numbers will be sampled wherever we are sampling from a probability distribution such as in lead time, patient weight, forecast quantity etc. These runs are called replications. Over several such runs we can analyse any metric that we are interested in, such as the service level. We can observe the mean value and the spread of this metric. In order to make the confidence interval smaller we need to run more replications.

We already discussed that the parameters in this optimization are the safety quantity that need to be produced at a country level. This safety quantity is in terms of extra days of cover. Since the OptQuest optimizer is a meta-heuristic based optimization engine we also need to define the range in which the optimizer will search for the optimal value and the step size. For example, for a particular country, we want the optimizer to search the days of cover from 1 day to 5 days with a step size of 1 (or a non integer value for partial days of cover). This means that for this country the optimizer has choice of 1,2,3,4 or 5. Each time the optimizer fixes the values of these parameters, we call it an iteration. The number of iterations and the number of replications to be run during optimization are the hyper parameters that can be configured from the user cockpit. We found in our case 100 replications and 500 iterations lead to a good solution. This number was arrived at using a trial and error approach. It is important to understand that the optimizer chooses a different set of parameters for each iteration and for each iteration (with the same value of the parameters) multiple replications are run. The overall approach is summarized in Figure 3.

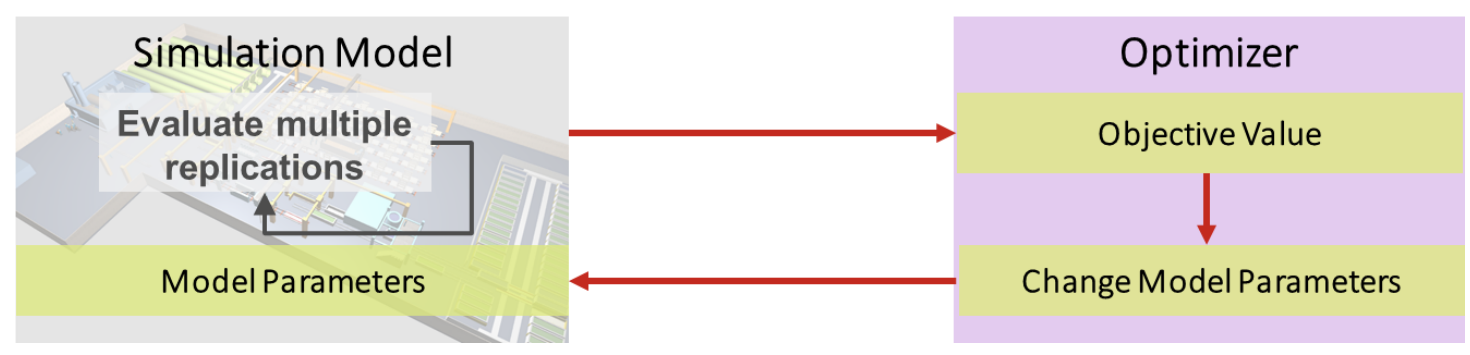

Figure 4 Simulation based optimization approach 
Since the total number of countries is large, we do not explicitly optimize for all the countries. Using the pareto principle (also known as the 80/20 rule) we arrived at eight countries that cover $80 \%$ of the demand. We optimize the days of cover for these eight countries and we use a ninth parameter which represents the days of cover in all other countries. In total we had nine parameters to be optimized. This way the problem was made tractable in an acceptable amount of time.

\section{RESULTS AND FUTURE SCOPE}

The most important result from our decision support system is shown in Figure 4. We show here the output of the simulation-based optimization experiment with 100 iterations, where each iteration has 100 replications. Each dot represents a single iteration with the values of the parameters selected by the optimizer. The $y$-axis shows the service level and the $\mathrm{x}$-axis shows the number of medicines scrapped for that iteration. While the optimizer does output the best iteration among all the iterations, we found it was better to select the best iteration visually by looking the plot in Figure 5. Each iteration will have 100 values for service level and corresponding scrap count as we run 100 replications. Hence each iteration will not be a single point but will have a spread in service level as well as in scrap count. We have shown the average values across the replications without the spread in Figure 4 and with spread of service level in Figure 5. There will also be a spread in the write-offs which is not shown here. This is one major reason that we select the best iteration visually. We can discard any iterations that are not robust (have a high spread of either service level or scrap count or both).

Through our improved production policy we could show a reduction in scrapped medicine by $28 \%$ as compared to the current performance without impacting the service leve1 as shown in Figure 4.

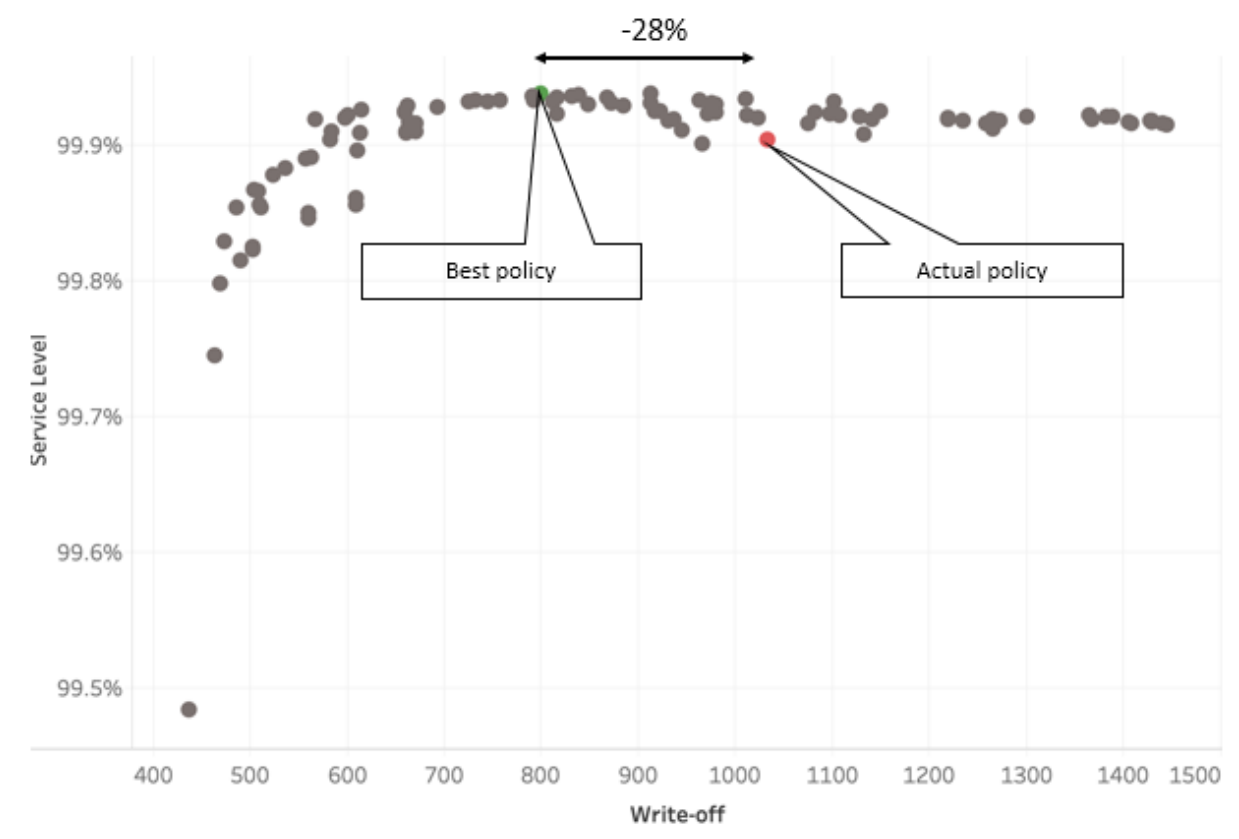

Figure 5 Trade-off between service level and write-offs 


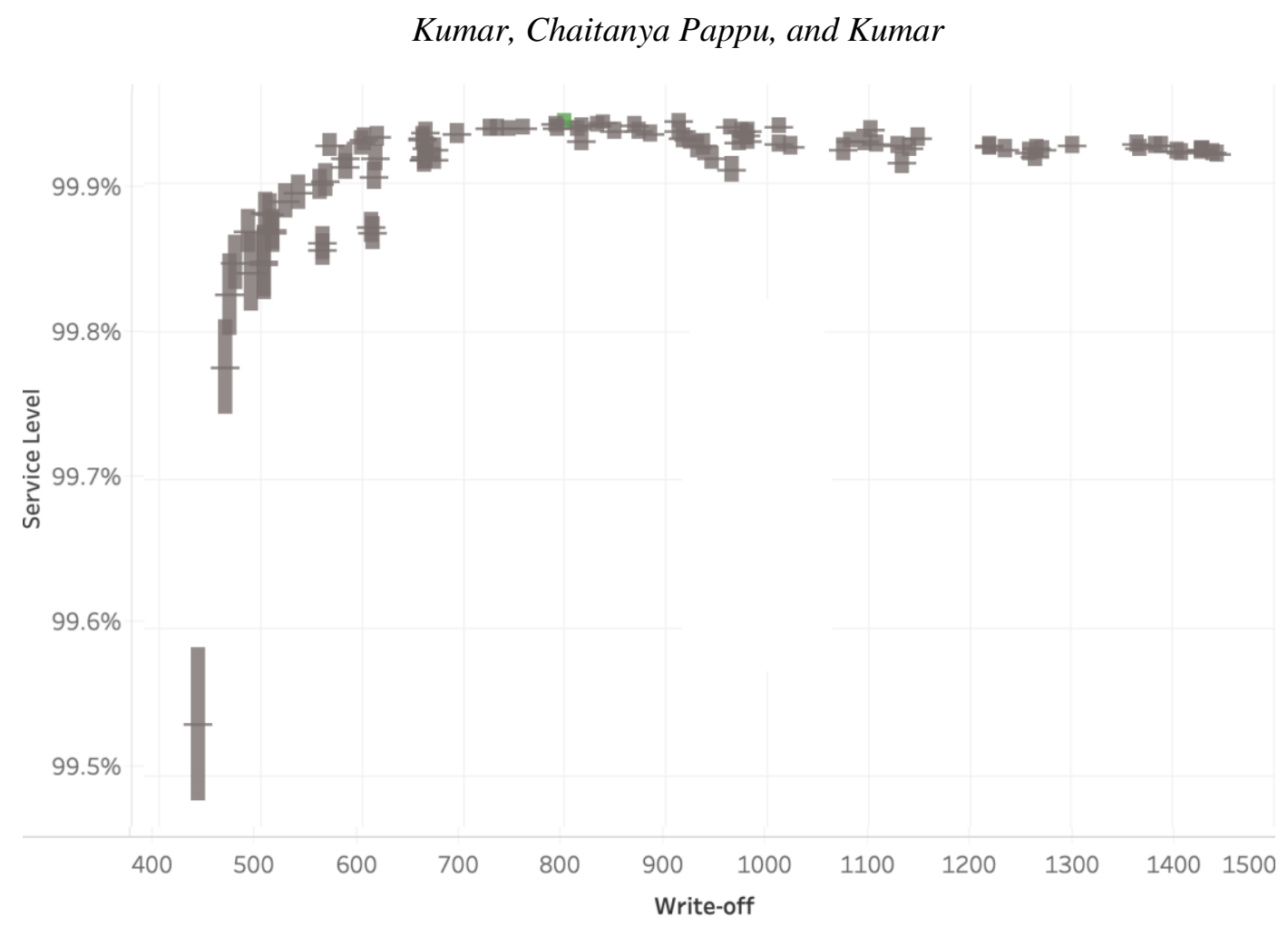

Figure 6 Trade-off between service level and write-offs with variation in service level

Once we have a simulation model of the supply chain created, we can try multiple ideas or scenarios to find an option that reduces cost or adds value in any other way. One of the scenarios we tried was to see the impact of lead time reduction. Another scenario tried was to delay the manufacturing a day. The current operations have a gap of 1 or 2 days between the date of manufactuing and date of shipment to cater to any delays/batch failures. A scenario of delaying the manufacturing by a day would increase the freshness of the batch on the day of treament there by enabling the ability to serve heavier patients with lesser number of medicines than earlier. One more scenario of potential saving by forecast bias reduction was tried. There are many countries which overforecast the demand just to avoid any stock outs.

For future work or further improvement in results we believe that in addition to using an optimization based approach it is possible to train a reinforcement learning based AI to make the decision of how much to produce. There has always been an interest in combining simulation with Artificial Intelligence(AI) techniques (Robert M et.al., 1987 and S Robinson et.al., 2005). With the latest infrastructure provided by companies such as Microsoft Bonsai as well as PathMind we feel that this approach should also be explored.

\section{REFERENCES}

Accorsi, R., Gallo, A., \& Manzini, R. (2017). A climate driven decision-support model for the distribution of perishable products. Journal of Cleaner Production, 165, 917-929.

Borshchev A and Grigoryev I (2020). The Big Book of Simulation Modeling: Multimethod Modeling with Anylogic 8. Lightning Source Inc.

C. Almeder and M. Preusser (2007). A toolbox for simulation-based optimization of supply chains, Proceedings of the 2007 Winter Simulation Conference, Washington, DC, 2007, pp. 1932-1939, doi: 10.1109/WSC.2007.4419821

C. Kouki, E. Sahin, Z. Jemai, Y \& Dallery (2013). Assessing the impact of perishability and the use of time temperature technologies on inventory management, International Journal of Production Economics, Volume 143, Issue 1, Pages 72-85 
Muhammed Ordu, Eren Demir, Chris Tofallis \& Murat M. Gunal (2020). A novel healthcare resource allocation decision support tool: A forecasting-simulation-optimization approach, Journal of the Operational Research Society, DOI: 10.1080/01605682.2019.1700186

OptQuest Optimization of Complex Systems, https://www.opttek.com/sites/default/files/pdfs/OptQuestOptimization\%20 of\%20Complex\%20Systems.pdf accessed on 03/09/2020

Pedro Amorim, Carlos H. Antunes \& Bernardo Almada-Lobo (2011). Multi-Objective Lot-Sizing and Scheduling Dealing with Perishability Issues, Industrial \& Engineering Chemistry Research 50(6), DOI: 10.1021/ie101645h

Robert M. O'Keefe \& John W. Roach (1987). Artificial Intelligence Approaches to Simulation, Journal of the Operational Research Society, 38:8, 713-722, DOI: 10.1057/jors.1987.120

S Robinson, T Alifantis, J S Edwards, J Ladbrook \& A Waller (2005). Knowledge-based improvement: simulation and artificial intelligence for identifying and improving human decision-making in an operations system, Journal of the Operational Research Society, 56:8, 912921, DOI: 10.1057/palgrave.jors.2601915

S. Nahmias (1982). Perishable inventory theory: A review, Operations Research, vol. 30, no. 4, pp. 680-707, 1982

QinglinDuanT. WarrenLiao (2013). A new age-based replenishment policy for supply chain inventory optimization of highly perishable products, International Journal of Production Economics, Volume 145, Issue 2, Pages 658-671

Xiaojun Wang, Dong Li (2012), A dynamic product quality evaluation based pricing model for perishable food supply chains, Omega, Volume 40, Issue 6, Pages 906-917

Yared Lemma, Daniel Kitaw \& Gulelat Gatew (2014). Loss in Perishable Food Supply Chain: An Optimization Approach Literature Review, International Journal of Scientific \& Engineering Research, Volume 5, Issue 5

Zhaotong Lian \& Liming Liu (2001). Continuous Review Perishable Inventory Systems: Models and Heuristics, IIE Transactions volume 33, pages 809-822

\section{AUTHOR BIOGRAPHIES}

AMIT KUMAR received a BTech in Electronics and Instrumentation Engineering from National Institute of Technology, Rourkela, India in 2008. He completed his Masters in Industrial Engineering from National Institute of Industrial Engineering (NITIE), Mumbai in 2012. He currently works as a principal consultant at ITC Infotech in Bangalore, India. https://www.linkedin.com/in/amit-kumar$71 \mathrm{~b} 8906 /$

KRISHNA CHAITANYA holds a Bachelor's in mechanical engineering from Osmania University, Hyderabad, India and Masters in Industrial Engineering from National Institute of Industrial Engineering, Mumbai India. He is currently working as principal consultant in ITC Infotech, Bangalore, India.

SUMIT KUMAR leads the Digital Solutions for manufacturing and supply chain at ITC Infotech, Bangalore, India. He has a BTech in Civil engineering from IIT Kharagpur and MBA in Operations Management from S.P. Jain Mumbai, India. 